\title{
Synthesis and maintenance of lipid droplets are essential for mouse preimplantation embryonic development
}

\section{$\operatorname{AUTHOR(S):~}$}

Aizawa, Ryutaro; Ibayashi, Megumi; Tatsumi, Takayuki; Yamamoto, Atsushi; Kokubo, Toshiaki; Miyasaka, Naoyuki; Sato, Ken; Ikeda, Shuntaro; Minami, Naojiro; Tsukamoto, Satoshi

\section{CITATION:}

Aizawa, Ryutaro ... [et al]. Synthesis and maintenance of lipid droplets are essential for mouse preimplantation embryonic development. Development 2019, 146(22): dev181925.

\section{ISSUE DATE:}

2019-11-15

\section{URL:}

http://hdl.handle.net/2433/253693

\section{RIGHT:}

(C) 2019. Published by The Company of Biologists Ltd; 許諾条件に基づいて揭載していま す。; The full-text file will be made open to the public on 25 November 2020 in accordance with publisher's 'Terms and Conditions for Self-Archiving'. 


\title{
Synthesis and maintenance of lipid droplets are essential for mouse preimplantation embryonic development
}

\author{
Ryutaro Aizawa ${ }^{1}$, Megumi Ibayashi, ${ }^{1, *}$, Takayuki Tatsumi ${ }^{2}$, Atsushi Yamamoto ${ }^{3}$, Toshiaki Kokubo ${ }^{1}$, \\ Naoyuki Miyasaka ${ }^{2}$, Ken Sato ${ }^{4}$, Shuntaro kkeda $^{5}$, Naojiro Minami ${ }^{5}$ and Satoshi Tsukamoto ${ }^{1, \mp}$
}

\begin{abstract}
Lipid droplets (LDs), which are ubiquitous organelles consisting of a neutral lipid core coated with a phospholipid monolayer, play key roles in the regulation of cellular lipid metabolism. Although it is well known that mammalian oocytes and embryos contain LDs and that the amount of LDs varies among animal species, their physiological functions remain unclear. In this study, we have developed a method based on two-step centrifugation for efficient removal of almost all LDs from mouse MII oocytes (delipidation). We found that delipidated MII oocytes could be fertilized in vitro, and developed normally to the blastocyst stage even when the embryos were cultured in the absence of a fatty acid supply. LDs were newly synthesized and accumulated soon after delipidation, but chemical inhibition of long chain acyl-CoA synthetases (ACSLs) blocked this process, resulting in severe impairment of early embryonic development. Furthermore, we found that overabundance of LDs is detrimental to early embryonic development. Our findings demonstrate the importance of synthesis and maintenance of LDs, mediated in part by ACSL activity, during preimplantation embryonic development.
\end{abstract}

KEY WORDS: Lipid droplet, Delipidation, Oocyte, Embryo, Mouse

\section{INTRODUCTION}

Lipid droplets (LDs) are dynamic organelles with important roles in lipid metabolism. LDs consist largely of neutral lipids, predominantly triglyceride (TG) and cholesterol ester (CE), and provide substrates for energy production, membrane components and signaling lipids (Walther and Farese, 2012; Welte, 2015). LDs are thought to be formed de novo in the endoplasmic reticulum (ER) because the proteins required for neutral lipid synthesis and LD formation both reside in the ER (Brasaemle et al., 2004; Buhman et al., 2001). LD growth is a complex process requiring the activities of multiple enzymes (Pol et al., 2014). Mature LDs consist of a neutral lipid core coated with a phospholipid monolayer (TauchiSato et al., 2002) containing structural proteins [such as perilipins

${ }^{1}$ Laboratory Animal and Genome Sciences Section, National Institute for Quantum and Radiological Science and Technology, Anagawa, Inage-ku, Chiba 263-8555, Japan. ${ }^{2}$ Comprehensive Reproductive Medicine, Regulation of Internal

Environment and Reproduction, Graduate School, Tokyo Medical and Dental

University, Tokyo 113-8519, Japan. ${ }^{3}$ Department of Reproduction Center, Dokkyo Medical University, Koshigaya Hospital, Saitama 343-8555, Japan. ${ }^{4}$ Laboratory of Molecular Traffic, Institute for Molecular and Cellular Regulation, Gunma University, Maebashi, Gunma 371-8512, Japan. ${ }^{5}$ Laboratory of Reproductive Biology, Graduate School of Agriculture, Kyoto University, Kyoto 606-8502, Japan. *Present address: Kameda IVF Clinic Makuhari, Nakase Mihama-ku, Chiba, 261-8501, Japan.

†Author for correspondence (tsukamoto.satoshi@qst.go.jp)

(D) R.A., 0000-0002-5236-1639; T.T., 0000-0003-0467-1939; K.S., 0000-00021034-5091; S.T., 0000-0001-7063-8825

Received 26 June 2019; Accepted 23 October 2019
(Bickel et al., 2009)] that regulate LD function. In addition to their association with the ER, LDs also interact with several other organelles, including mitochondria, Golgi, peroxisomes and lysosomes in order to help the cell adapt to the nutrient environment (Valm et al., 2017). Although LDs have been observed in most cell types throughout the animal kingdom, their function is much broader than previously anticipated; LDs are not only important for energy storage and as sources of lipids for steroid hormone production, but also act as storage sites for nuclear proteins (Cermelli et al., 2006; Welte, 2015).

During mammalian oogenesis, LDs are stored in the cytoplasm, although for unknown reasons the exact content of LDs varies widely among species (Bradley and Swann, 2019; Dunning et al., 2014). Mouse oocytes/embryos store very few LDs relative to pig, which contain high levels of stored lipids [fatty acid lipid content: $25.7 \times 10^{-5} \mathrm{ng} / \mu \mathrm{m}^{3}$ in porcine oocytes versus $2.05 \times 10^{-5} \mathrm{ng} / \mu \mathrm{m}^{3}$ in mouse (Bradley and Swann, 2019)]. Because the level of LDs may reflect the extent of $\beta$-oxidation, fatty acids produced by lipolysis and/or lipophagy (Zechner et al., 2017) might be the primary energy source in porcine oocytes/embryos. By contrast, probably owing to the lower level of LDs, mouse oocytes/embryos have long been thought to use pyruvate, lactate and glucose as their principal energy substrates, although these substrates are strictly regulated during preimplantation development (Gardner and Leese, 1986; Leese and Barton, 1984). However, several lines of evidence suggest that LD biogenesis is physiologically important during early embryonic development: (1) LD morphology changes dynamically during embryonic development and in response to culture conditions (Bradley et al., 2016; Watanabe et al., 2010); (2) preimplantation embryos can take up exogenous FAs for storage as neutral lipid (mainly TG), and for use in $\beta$-oxidation (Hillman and Flynn, 1980); and (3) $\beta$-oxidation plays a vital role in the development of preimplantation embryos (Dunning et al., 2010).

To elucidate the roles of LDs, we recently developed a selective autophagy-based method, termed forced lipophagy, to achieve LD depletion (Tatsumi et al., 2018). Forced lipophagy relies on autophagic activity and expression of the autophagic adaptor p62, also known as Sqstm1 (Ichimura et al., 2008), on the surface of LDs. p62 facilitates the interaction of LDs with LC3, which stably binds to the autophagosomal membrane, forming a bridge between LDs and the membrane. LDs sequestered by the autophagosome ultimately fuse with the lysosome, where they are degraded. Using a forced lipophagy system, we could decrease LD size and number, as well as TG content. Under these conditions, $~ 50 \%$ of embryos were developmentally retarded until the blastocyst stage, suggesting that a proper level of LDs is essential for normal preimplantation embryonic development. Although forced lipophagy is a useful approach for studying LD function in living oocytes and embryos, one limitation is that the efficiency of forced lipophagy depends on intrinsic autophagic activity; consequently, 
not all LDs are eliminated. Therefore, in order to determine the precise function of LDs during early embryonic development, a more specific method that completely removes LDs from mouse oocytes and/or embryos is required.

In this study, we developed a delipidation method for efficiently removing LDs from mouse MII oocytes. Using the method, we found that, even after delipidation, MII oocytes could be fertilized and developed normally. Unexpectedly, we found that immediately after delipidation, LDs were newly synthesized and accumulated through induction of fatty acids mediated by acyl-CoA synthetase activity. These newly synthesized LDs, as well as their proper storage, were crucial for preimplantation embryonic development.

\section{RESULTS}

\section{Complete removal of LDs (delipidation) from MII oocytes by} two-step centrifugation

While delipidation based on centrifugation has been used frequently in porcine oocytes/embryos (Nagashima et al., 1995, 1994; Yoneda et al., 2004), no method has been previously developed for mouse. Hence, we sought to optimize a delipidation method for application to mouse oocytes and embryos.

To determine the most efficient delipidation condition, we collected MII oocytes from superovulated C57BL/6 females and performed an initial centrifugation at $4200 \mathrm{~g}$ for $10 \mathrm{~min}$ (Fig. 1A). We found that this centrifugation caused aggregation of LDs in the cell periphery (Fig. 1B, left). However, because the aggregated LDs remained tightly associated with cellular structures, it was difficult to remove them completely without also depleting other cytoplasmic contents, and the subsequent procedure (removal of aggregated LDs using a micropipette) tended to cause severe damage, leading to oocyte death (data not shown).

To overcome these problems, we centrifuged MII oocytes at $9500 \mathrm{~g}$ for $10 \mathrm{~min}$ under hyperosmolar conditions ( 440 mOsm). This manipulation increased the size of the perivitelline (PV) space, and the aggregated LDs were released into the larger PV space (Fig. 1A); a similar procedure has previously been demonstrated in porcine embryos (Men et al., 2015). Strikingly, these additional treatments led to the release of nearly all aggregated LDs into the PV space with less damage to the oocyte (Fig. 1B, right); more than $95 \%$ of MII oocytes survived after two-step centrifugation (Fig. 1C, left) and nearly $90 \%$ of their LDs were released into the PV space (Fig. 1C, right). In the final step, the released LDs were successfully isolated using a micropipette (Fig. 1D, Movie 1). We also verified that this method could be applied to MII oocytes, which were derived from different mouse strains, including B6 (used in this study), C3H and ICR (Fig. S1).

\section{Specific isolation of LDs without cytoplasmic contamination}

We confirmed that our method resulted in highly efficient removal of LDs from MII oocytes without extra cytoplasmic contamination.
A

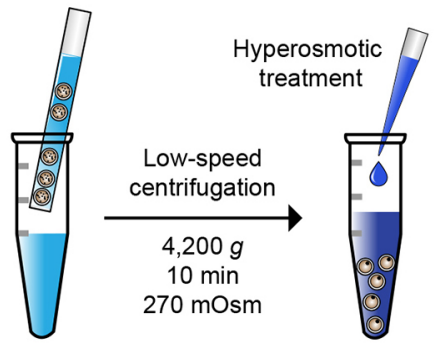

B

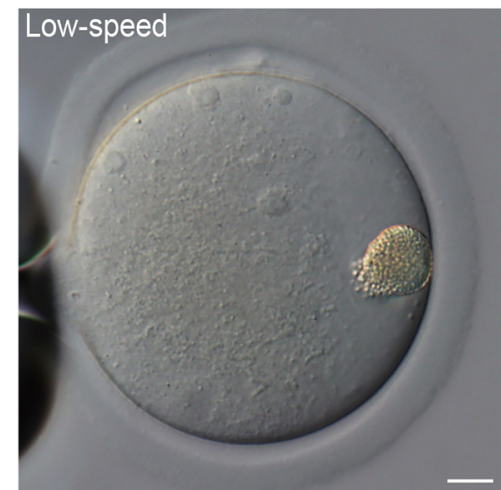

C

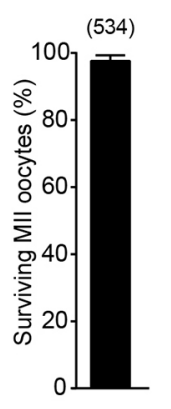

D

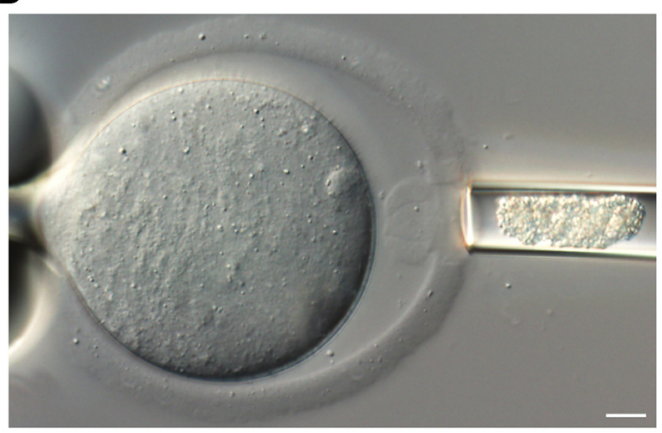

Fig. 1. Isolation of LDs from MII oocytes by two-step centrifugation. (A) Schematic representation of the delipidation method for mouse MII oocytes. MII oocytes were initially centrifuged at low speed (10 min at $4200 \mathrm{~g}$ ) to aggregate LDs in the cell periphery. After addition of hyperosmotic medium (630 $\mathrm{mOsm}$ ) to increase the perivitelline (PV) space, MIl oocytes were centrifuged at high speed (10 $\mathrm{min}$ at $9500 \mathrm{~g}$ ) to release the aggregated LDs into the PV space. (B) Representative images of MII oocytes after the first (low speed, left) and second (high speed, right) centrifugation. (C) Percentage of surviving MII oocytes (left) and the efficiency of LD release into the PV space (right) after two-step centrifugation. The total number of MII oocytes tested is shown in brackets above each bar. Data are mean \pm s.e.m. of five independent experiments. (D) Removal of LDs from the PV space using a micropipette. Scale bars: $10 \mu \mathrm{m}$ See also Movie 1. 
Staining of individual organelles with specific fluorescent dyes revealed that the ER, lysosome and mitochondria were retained in the cytoplasm even after the second centrifugation, whereas almost all LDs (which were labeled with the fluorescent neutral lipid dye BODIPY 493/503) were localized in the PV space (Fig. 2A). Consistent results were obtained by EM analysis: LDs were clustered and aggregated at the cell periphery after the first centrifugation (Fig. 2B, left), and were released into the PV space with very little accompanying cytoplasmic content after the second centrifugation (Fig. 2B, right). Furthermore, we performed LC-MS analysis to determine the level of TG species in non-delipidated or delipidated MII oocytes, and found that the levels of most TG species measured [primarily oleic (18:1), linoleic (18:2) and palmitic acids (16:0)] were significantly reduced after delipidation (Fig. 2C). Together, these results clearly demonstrate that LDs can be specifically removed from the cytoplasm of mouse MII oocytes by two-step centrifugation combined with hyperosmotic treatment.

\section{Delipidated MII oocytes were fertilized and developed normally}

Next, to determine whether LD depletion would influence the developmental potential of MII oocytes, we performed in vitro fertilization (IVF) or intracytoplasmic sperm injection (ICSI), and analyzed the subsequent development of the embryos. The results revealed that although the IVF rate of delipidated MII oocytes was significantly reduced to about $50 \%$ of the level in non-delipidated MII oocytes (Fig. 3A, left; $P<0.01$, Student's $t$-test), MII oocytes could be efficiently fertilized by ICSI despite delipidation (Fig. 3A, right; $P=0.221$, Student's $t$-test). These results indicate that delipidation, especially a two-step centrifugation or hyperosmotic treatment, might affect the zona pellucida (ZP), thus preventing sperm-egg interactions. We found that ZP-free delipidated MII oocytes were efficiently fertilized by IVF relative to ZP-free non-delipidated MII oocytes (Fig. 3B, left, $P=0.158$, Student's $t$-test). Consistent with this, ZP drilling greatly improved IVF efficiency (Fig. 3B, right, $P=0.453$, Student's $t$-test). These results support our prediction that changing the characteristics of $\mathrm{ZP}$, but not the plasma membrane, during the delipidation procedure is responsible for the lower IVF success rate. However, once the delipidated MII oocytes were fertilized by IVF or ICSI, their subsequent developmental potential was comparable with that of non-delipidated MII oocytes, even when the embryos were cultured in the absence of an extracellular FA supply (Fig. 3C,D and Movie 2).
A

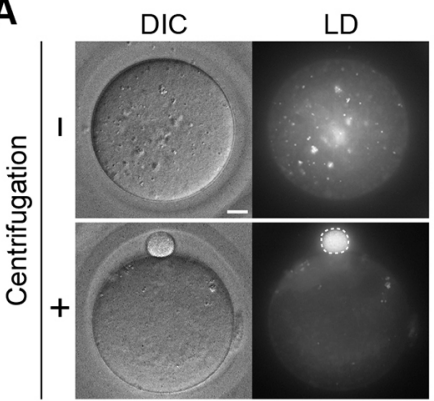

B

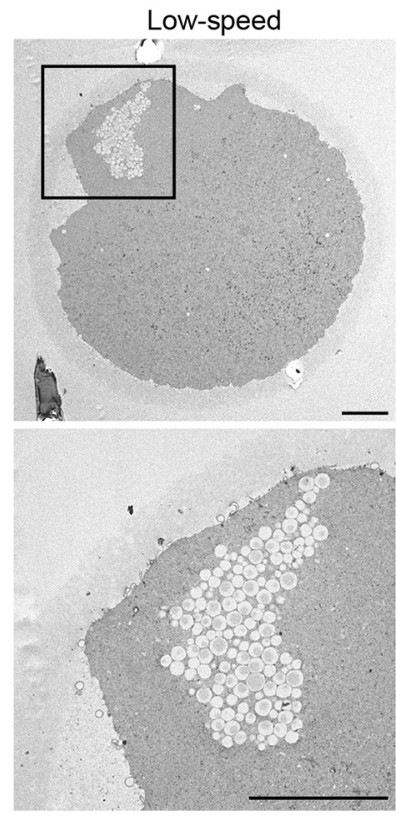

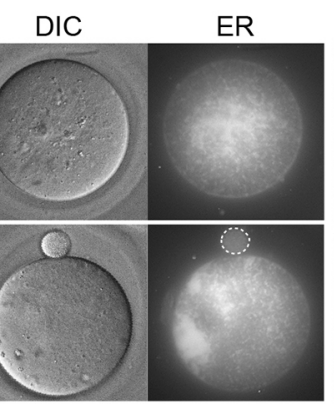

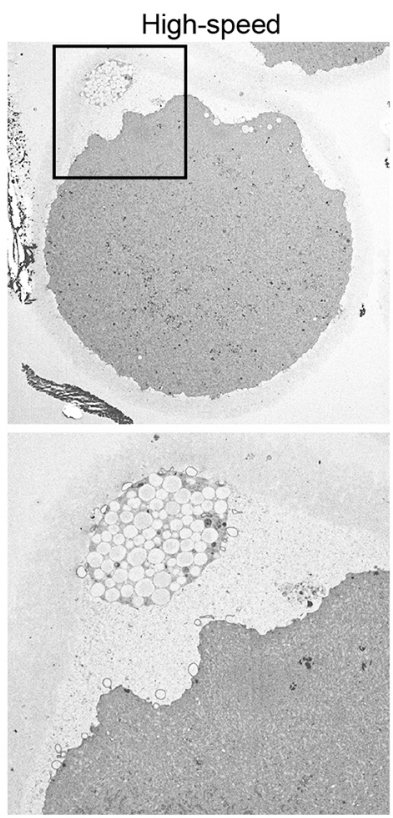

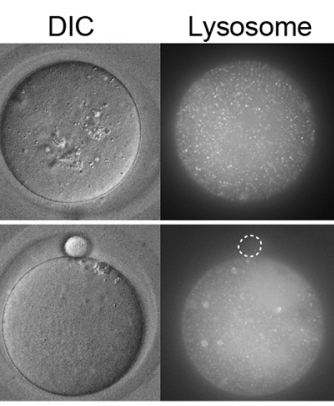

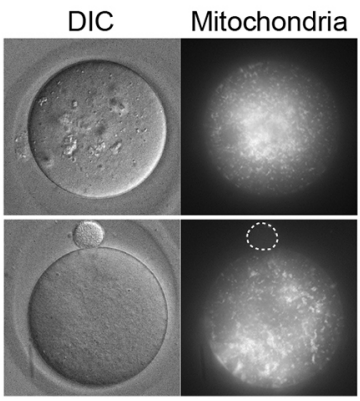

C

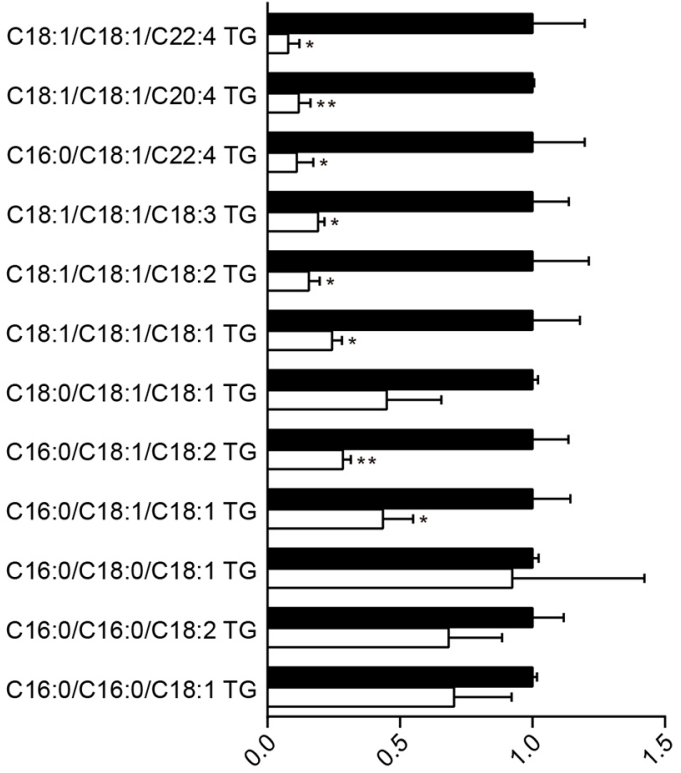

Fig. 2. Specific isolation of LDs by the delipidation method. (A) MIl oocytes before (top) and after (bottom) two-step centrifugation were stained with organelle-specific dyes and observed by confocal fluorescence microscopy. Dashed lines outline LDs in the PV space. DIC, differential interference contrast. (B) EM images of MIl oocytes after the first (low speed, left) and second (high speed, right) centrifugation. Bottom panels show higher-magnification images of the representative boxed area. (C) Quantification showing relative levels of TG species measured by LC-MS in non-delipidated (non-DeLD) or delipidated (DeLD) MII oocytes. Data are mean \pm s.e.m. of three independent experiments with $100 \mathrm{Mll}$ oocytes in each condition. ${ }^{*} P<0.05,{ }^{* *} P<0.01$, Student's $t$-test. Scale bars: $10 \mu \mathrm{m}$. 
A

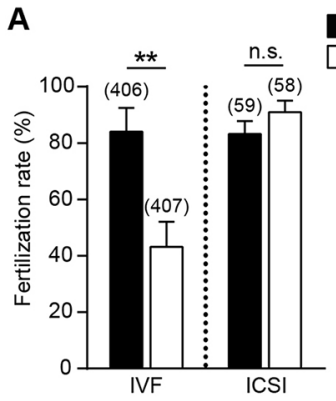

C

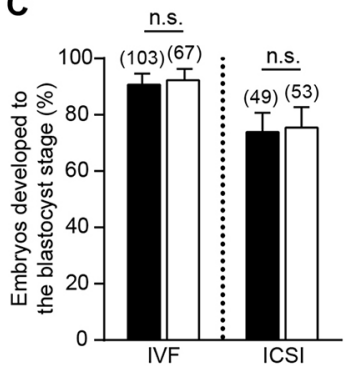

B

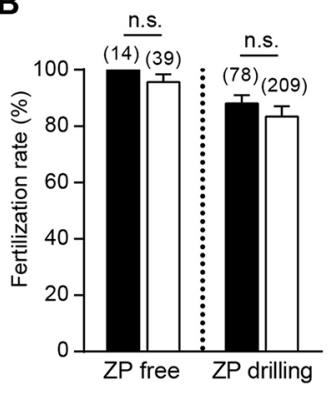

D

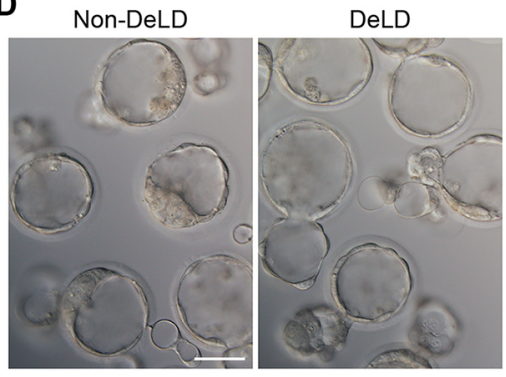

Fig. 3. Developmental potential of delipidated MII oocytes. (A) Percentage of successful fertilization in non-delipidated (non-DeLD) or delipidated (DeLD) MII oocytes, which were fertilized by IVF (left) or ICSI (right). (B) Percentage of successful fertilization in ZP-free (left) or ZP-drilled (right) MII oocytes. MII oocytes with or without delipidation were treated with acidic Tyrode's solution or drilled, and the resultant MII oocytes were fertilized by IVF. (C) Percentages of embryos developing into blastocysts. Non-DeLD or DeLD MII oocytes were fertilized by IVF (left) or ICSI (right); fertilized embryos were selected and cultured until the blastocyst stage. (D) Representative images of blastocysts, developed from non-DeLD or DeLD MII oocytes, fertilized by IVF and cultured in FA-free KSOM medium for 4 days. The rate of successful fertilization was evaluated based on the presence of two pronuclei and a second polar body $(A, B)$. Number of MIl oocytes/embryos analyzed is specified in brackets above each bar. Data are from four to seven independent experiments, and are mean \pm s.e.m. n.s., non-significant, ${ }^{* *} P<0.01$, Student's $t$-test. Scale bar: $50 \mu \mathrm{m}$. See also Movie 2.

\section{LDs are newly synthesized immediately after delipidation}

The fact that delipidation did not affect developmental potential implied that LDs stored in MII oocytes may be dispensable. However, when observing the delipidated MII oocytes/embryos in more detail, we noticed that a few small LDs, possibly representing newly forming LDs, appeared soon after delipidation (Fig. 4A, left panel, MII). The number of new small LDs increased over the course of embryonic development (Fig. 4A, middle to right; one- to two-cell stage). As a result, LD distribution became similar to that of non-delipidated embryos, likely owing to the continuous production of new LDs.

Consistent results were obtained by electron microscopy (EM): non-delipidated MII oocytes contained a large number of clustered LDs, and numerous LDs were observed at the two-cell stage (Fig. 4B, top), in accordance with earlier findings (Bradley et al., 2016). In contrast, delipidated MII oocytes contained very few LDs, but many small LDs appeared at the two-cell stage (Fig. 4B, bottom). Quantitative analysis revealed a significant decrease in both LD area (16.7\%) and LD number (12.8\%) in delipidated MII oocytes relative to those in non-delipidated MII oocytes (Fig. 4C), whereas at the two-cell stage, LD area and number were $50.0 \%$ and $54.4 \%$, respectively, relative to non-delipidated two-cell embryos (Fig. 4C).

The increase in abundance of new LDs was also observed when the delipidated MII oocytes were cultured in BSA-free medium

(FHM supplemented with PVP instead of FA-free BSA, Fig. S2), indicating that new LD synthesis occurs regardless of the presence of albumin, which acts as an FA carrier. Based on these results, we presumed that these newly synthesized LDs might contribute to normal embryonic development after delipidation.

To exclude the possibility that newly synthesized LDs were due to growth of pre-existing (previously synthesized) LDs, we sought to visualize the formation and subsequent growth of new LDs. For this purpose, we used BODIPY 558/568 C12 (BODIPY-C12) combined with BODIPY 493/503. BODIPY-C12 is a widely used fluorescent long-chain FA analogue consisting of a 12-carbon saturated FA bound to the BODIPY fluorophore at its hydrophobic end, which is esterified within the cell and incorporated into LD-specific neutral lipids, allowing visualization of the earliest steps of FA-derived LD formation (Kassan et al., 2013; Rambold et al., 2015). Non-delipidated or delipidated MII oocytes were incubated with BODIPY-C12 followed by BODIPY 493/503 staining, and were then observed by confocal fluorescence microscopy. We found that the number of small structures labeled by BODIPY-C12 were remarkably increased in delipidated MII oocytes, whereas such structures were less prominent in nondelipidated MII oocytes (Fig. 4D, top). Importantly, not all structures were detectable with BODIPY 493/503: more of the larger structures were preferentially labeled using BODIPY 493/503, presumably because nascent (small) LDs had very low lipid mass and were therefore difficult to detect using this labeling method. These features indicate that these abundant small structures represent newly forming LDs. Consistent results were obtained at the two-cell stage: most of the LDs, labeled using BODIPY-C12, were clearly visible and hence detectable using BODIPY 493/503 (Fig. 4D, bottom), suggesting that they are mature LDs. In addition, time-lapse imaging of delipidated MII oocytes revealed that BODIPY-C12 fluorescence, which initially appeared in a reticular pattern resembling the ER (Fig. S3A), subsequently accumulated in tiny puncta (likely representing newly LDs) and ultimately became detectable using BODIPY 493/503 (Fig. S3B). Taken together, these findings indicate that LD biogenesis is highly activated after delipidation.

\section{Regulation of LD content through ACSL activity is important for embryonic development}

$\mathrm{LD}$ formation is a stepwise process that requires multiple enzymatic activities (Pol et al., 2014). Because free FAs are chemically inert, once inside the cell, they must be activated by esterification by acylCoA synthetases. Several different types of acyl-CoA synthetase exist. The long-chain acyl-CoA synthetase (ACSL) proteins activate long-chain fatty acids to acyl-CoA, which are then used for the synthesis of esterified lipids, such as TG and CE. There are five different mammalian isoforms of the ACSL family (ACSL1, 3, 4, 5 and 6) (Mashek et al., 2004), which have different FA preferences and tissue specificity of expression (Fujino et al., 1996; Kang et al., 1997). Among them, ACSL3 is found in growing and mature LDs (Fujimoto et al., 2004; Kassan et al., 2013). Inhibition of this enzymatic activity using triacsin C (Tomoda et al., 1987) greatly reduces TG synthesis (Igal and Coleman, 1996), and thereby blocks FA-induced formation of LDs (Herms et al., 2013; Kassan et al., 2013).

We hypothesized that the newly synthesized LDs observed soon after delipidation might be catalyzed by ACSLs activity. To explore this possibility, we fertilized delipidated MII oocytes, co-cultured them with triacsin C, and stained them using BODIPY 493/503. In triacsin C-treated embryos, LDs labeled with BODIPY 493/503 were significantly less abundant in two-cell embryos, and very few LDs were detectable at the four-cell stage, whereas no obvious 
A

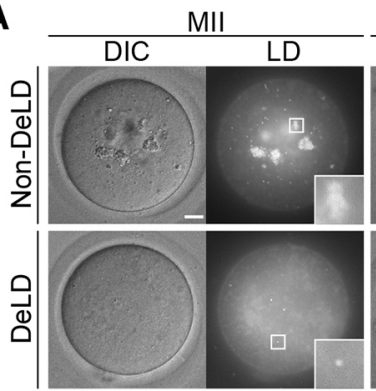

B

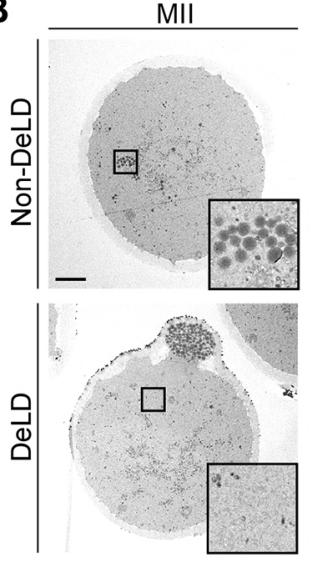

D

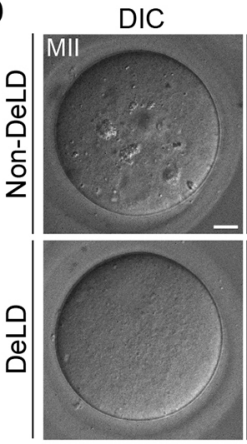

BODIPY-C12

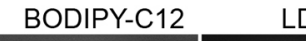

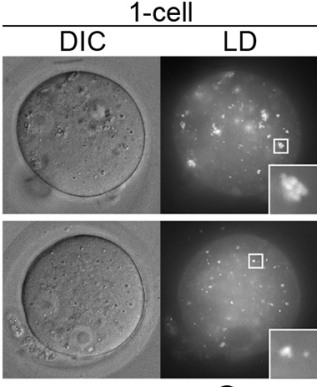

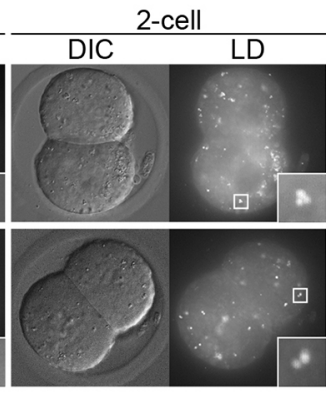

2-cell

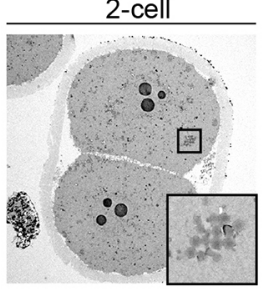

C $\widetilde{\widetilde{\xi}}^{2.0}$

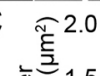

Fig. 4. New synthesis of LDs after delipidation. (A) Nondelipidated (non-DeLD) or delipidated (DeLD) MII oocytes or fertilized embryos at the indicated stages were stained with BODIPY 493/503 and observed by confocal fluorescence microscopy. (B) EM images of non-DeLD or DeLD MII oocytes or two-cell embryos. (C) Quantification of LD area (top) and number (bottom) in non-DeLD or DeLD MII oocytes and two-cell embryos. $n$, number of Mll oocytes or two-cell embryos analyzed. ${ }^{* *} P<0.01,{ }^{* *} P<0.001$, Mann-Whitney $U$-test. Data are mean \pm s.e.m. (D) Non-DeLD or DeLD MII oocytes, or twocell embryos were stained with both BODIPY-C12 (red) and BODIPY 493/503 (green). Red arrows indicate BODIPY-C12positive LDs; green arrows indicate BODIPY-C12 and BODIPY 493/503 double-positive LDs. Insets show higher-magnification images of the representative boxed areas. DIC, differential interference contrast. Scale bars: $10 \mu \mathrm{m}$.

changes were detected in DMSO-treated embryos (Fig. 5A, right panels). Consistent with this observation, quantification of the BODIPY 493/503 signal in delipidated embryos revealed that the area occupied by LDs at the two-cell and four-cell stage was $72.9 \%$ and $86.9 \%$ lower, respectively, than in DMSO-treated embryos (white bars in Fig. 5B), indicating that triacsin C specifically inhibits new LD formation. Analysis of the developmental potential of these embryos revealed that their development was normal up to the eightcell stage. However, all the embryos failed to develop further, subsequently fragmented or died around the morula stage. By contrast, delipidated embryos co-cultured with DMSO continued to develop to the blastocyst stage (Fig. 5C,D and Movie 3).
We assumed that triacsin $\mathrm{C}$ would have no effect on the development of non-delipidated embryos, as they contain preexisting LDs and consequently have no need to re-synthesize LDs. Unexpectedly, however, we found that triacsin $\mathrm{C}$ caused a marked reduction in the number of preexisting LDs over time (Fig. 5A, left panels). Consistent with this, quantification of the BODIPY 493/ 503 signal in non-delipidated embryos demonstrated that the total LD areas at the two- and four-cell stages decreased 78.1\% and $74.9 \%$, respectively, relative to DMSO-treated embryos (black bars in Fig. 5B). As a result, triacsin $\mathrm{C}$ treatment caused developmental arrest before the morula stage, a result similar to that observed in the delipidated embryos (Fig. 5C,D and Movie 3). 
A
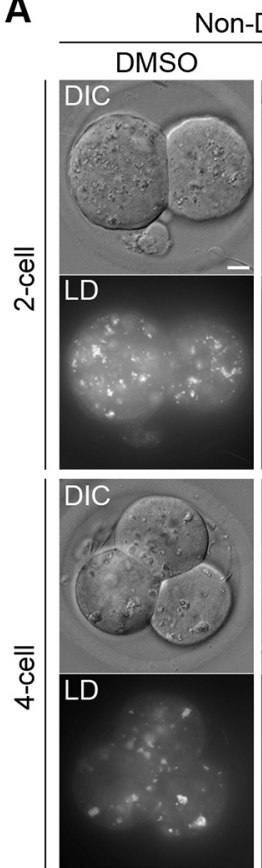

Non-DeLD
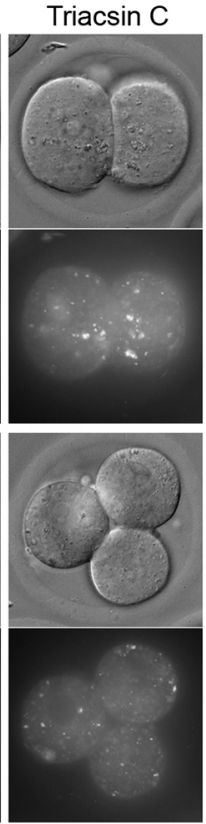

DeLD
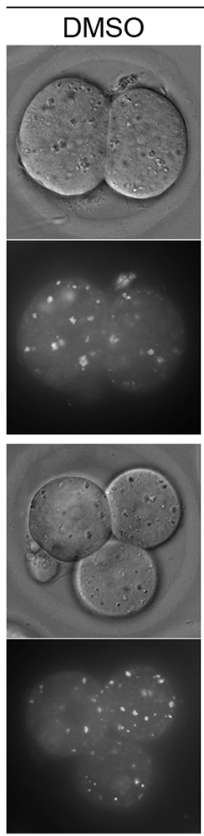

B

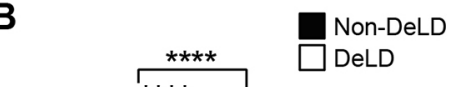

C
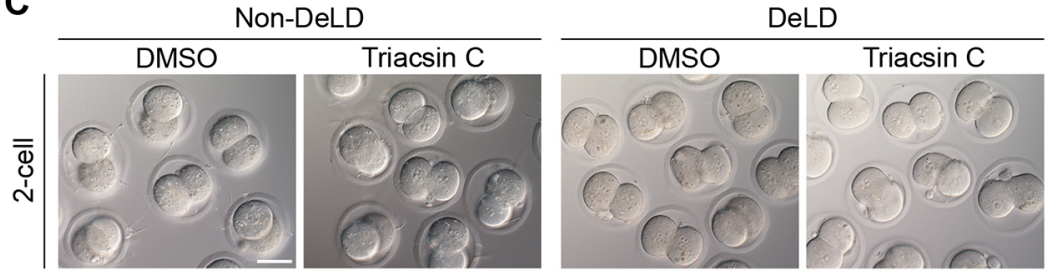

D
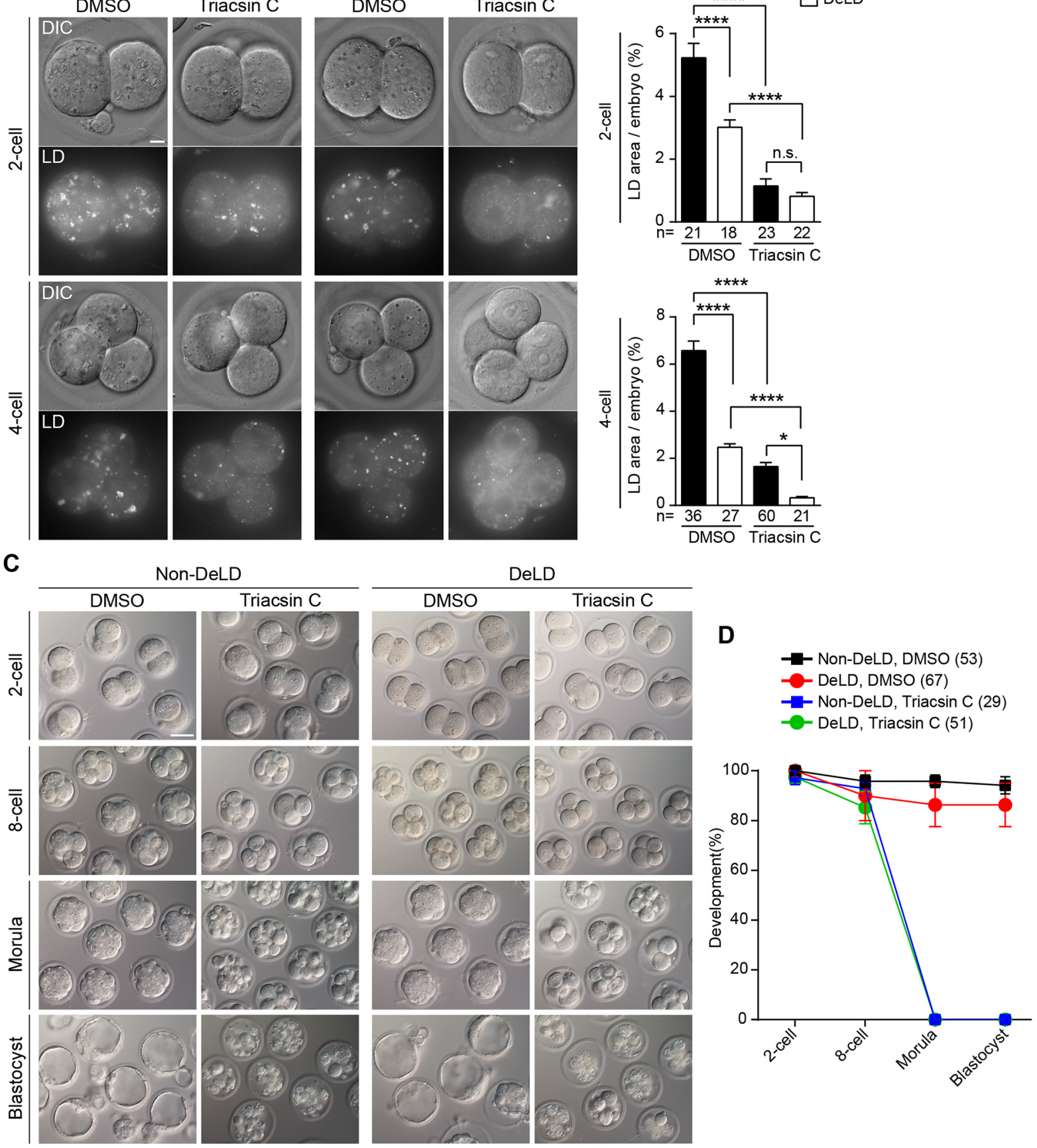

Fig. 5. The importance of LD synthesis and LD maintenance through ACSL activity during preimplantation embryonic development. (A) Fertilized embryos, developed from non-delipidated (non-DeLD) or delipidated (DeLD) MII oocytes, were cultured in the presence of DMSO or triacsin C and stained with BODIPY 493/503 at the indicated stages. (B) Quantification of total LD area in two-cell (top) and four-cell (bottom) embryos with the indicated treatment. $n$, numbers of embryos analyzed. n.s., non-significant, ${ }^{*} P<0.05,{ }^{* \star \star *} P<0.0001$, Mann-Whitney U-test. Data are mean \pm s.e.m. (C) Preimplantation development of embryos, developed from non-DeLD or DeLD MII oocytes, which were fertilized by IVF and co-cultured in the presence of DMSO or triacsin C. (D) Percentages of embryos reaching each developmental stage. Non-DeLD or DeLD MII oocytes were fertilized by IVF and co-cultured in the presence of DMSO or triacsin C. Data are mean \pm s.d. of more than three independent experiments. Number of embryos analyzed is specified in brackets. Treatment of embryos with DMSO was used as a control. DIC, differential interference contrast. Scale bars: $10 \mu \mathrm{m}$ in A; $50 \mu \mathrm{m}$ in C. See also Movie 3.

These results imply that cellular lipolysis activity (i.e. lipase activity) is exceptionally high, facilitating the degradation of pre-existing LDs and leading to LD depletion, explaining why non-delipidated embryos did not develop beyond the eight-cell stage. An alternative possibility is that ACSL activity is required not only for new LD synthesis but also for their storage, as has been demonstrated in mammalian cultured cells (Fujimoto et al., 2007).

At the concentration used in this study, triacsin $\mathrm{C}$ is highly specific for ACSL1, ACSL3 and ACSL4, but does not inhibit ACSL5 or ACSL6 (Vessey et al., 2004), and our quantitative RT-PCR analysis of non-delipidated or delipidated MII oocytes revealed that the level 
of Acsl3 mRNA was higher than the levels of Acsl1, Acsl4, Acsl5 and Acsl6 mRNAs (Fig. S4). These observations imply that ACSL activity in oocytes and/or early embryos is highly dependent on ACSL3.

Although we cannot exclude the possibility that triacsin $\mathrm{C}$ plays a role beyond LD biogenesis, our results suggest that ACSL activity has an important function not only in new LD synthesis but also in their maintenance during preimplantation embryonic development.

\section{An overabundance of LDs influences embryonic development}

Our results indicate that an imbalance between LD synthesis and use (i.e. LD overabundance) influences early embryonic development.
To directly demonstrate the impact of LD overabundance in early embryonic development, we isolated LDs from MII oocytes and transplanted them into the individual blastomeres of two-cell embryos, which were developed from non-delipidated or delipidated MII oocytes (Fig. 6A and Movie 4), and examined their preimplantation development. However, such LD-transplanted embryos developed normally to the blastocyst stage despite delipidation (Fig. 6B).

We presumed that transplanted LDs might be degraded by cellular lipases, which contribute in this manner to normal embryonic development in LD-transplanted embryos. To test this possibility, we cultured LD-transplanted embryos with the lipase inhibitor diethylumbelliferyl phosphate (DEUP). As we expected,
A

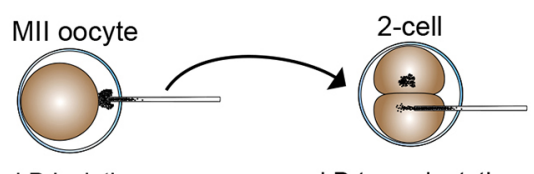

LD isolation
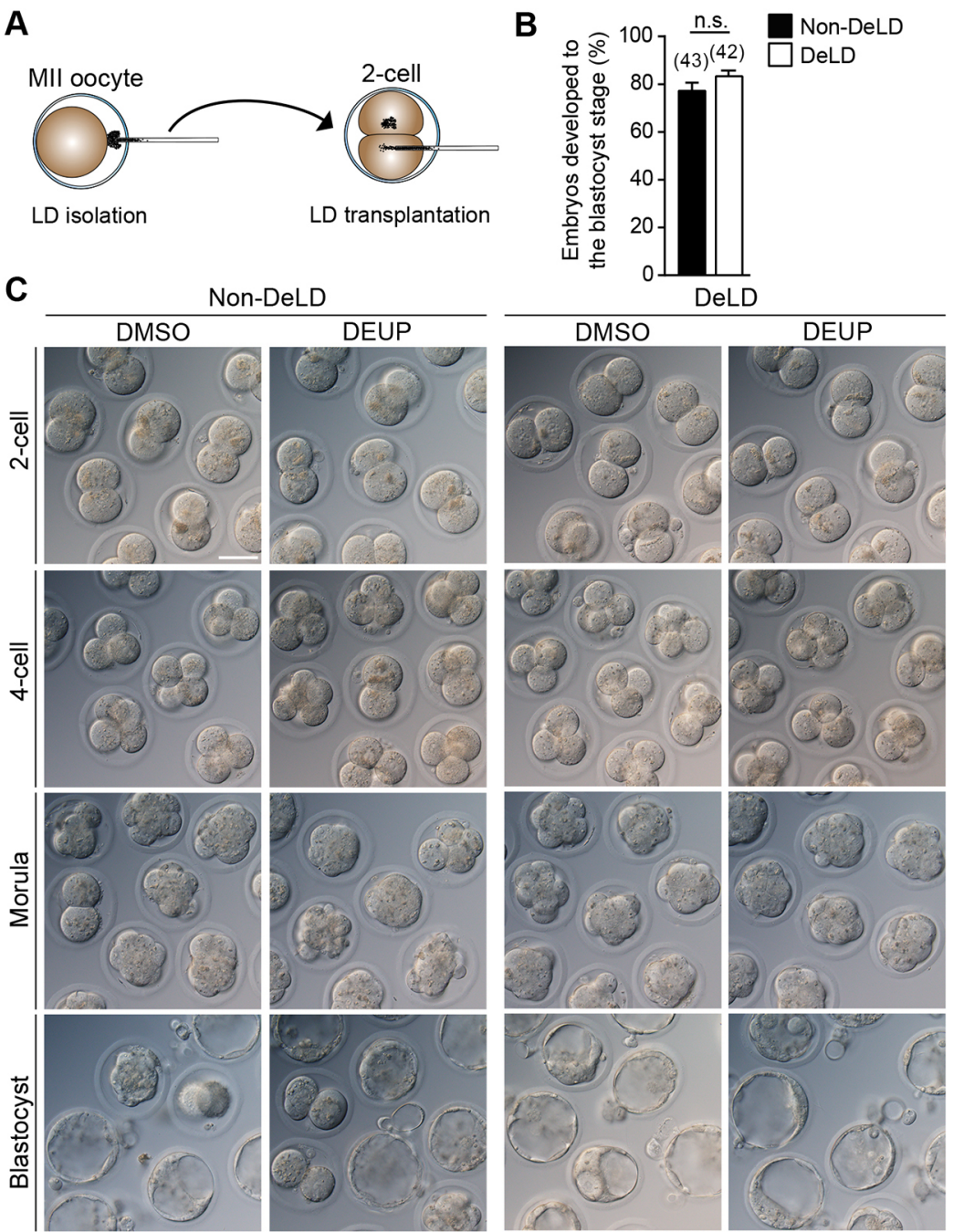

D

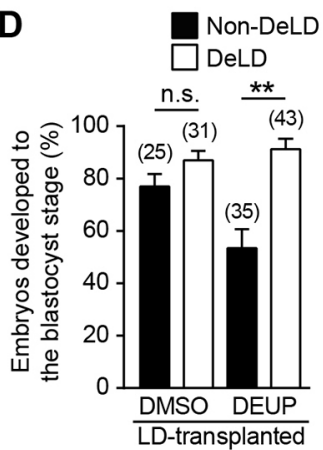

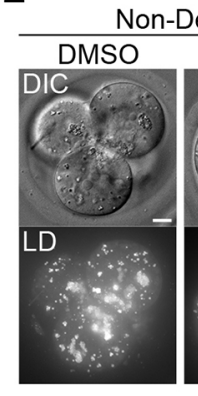

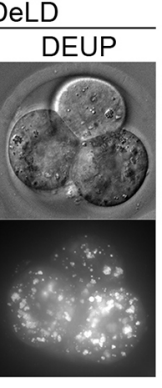

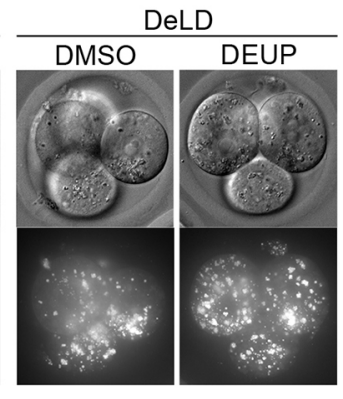

Fig. 6. The effect of LD overabundance on early embryonic development. (A) Schematic illustration of LD transplantation. LDs isolated from MII oocytes were divided into two pieces, and each LD was transplanted into individual blastomeres of two-cell embryos. See also Movie 4. (B) Percentages of LD-transplanted embryos, which were developed from non-delipidated (nonDeLD) or delipidated (DeLD) MII oocytes, reaching the blastocyst stage. Number of LD-transplanted embryos is specified in brackets above the bar. Data are from four independent experiments, and are mean \pm s.e.m. n.s., nonsignificant, Student's t-test. (C) Preimplantation development of LD-transplanted embryos. LDs isolated from MII oocytes were transplanted into individual blastomeres of two-cell embryos, which were developed from non-DeLD or DeLD MII oocytes, and the resultant embryos were cultured in the presence of DMSO or DEUP. (D) Percentage of embryos reaching each developmental stage under the indicated conditions. DMSOtreated embryos were used as a control. Data are mean \pm s.d. of three independent experiments. n.s., non-significant, ${ }^{*} P<0.01$, Student's t-test. (E) LD-transplanted embryos developed from non-DeLD or DeLD were cultured in the presence of DMSO or DEUP, stained with BODIPY 493/503 at the four-cell stage and observed by confocal fluorescence microscopy. Images are maximum projections of the $z$ sections ( 10 sections, $2 \mu \mathrm{m}$ steps). Number of embryos analyzed is specified in brackets above the bar. DIC, differential interference contrast. Scale bars: $50 \mu \mathrm{m}$ in $\mathrm{C} ; 10 \mu \mathrm{m}$ in $\mathrm{E}$. 
the proportion of embryos that reached the blastocyst stage was substantially lower in non-delipidated embryos $(53.4 \%)$ than in delipidated embryos $(91.2 \%$, Fig. 6C,D). By contrast, the developmental rate was not significantly changed when the nondelipidated or delipidated embryos were cultured with DMSO (as a control) following LD transplantation $(77.0 \%$ in non-delipidated embryos versus $87.0 \%$ in delipidated embryos, Fig. 6C,D). BODIPY 493/503 staining revealed that DEUP treatment caused substantial LD accumulation in these LD-transplanted embryos relative to DMSO-treated embryos (Fig. 6E), indicating that these effects were specific for lipase activity. Similar results were obtained when these LD-transplanted embryos were cultured with another lipase inhibitor (atglistatin, data not shown). Together, our results support the prediction that cellular lipase activity contributes at least in part to degradation of excess LDs in preimplantation embryos. The results also indicate that LD overabundance influences early embryonic development.

Finally, we sought to rescue the triacsin C-induced developmental defect by LD transplantation. However, LD transplantation was not sufficient to rescue the embryonic impairment: neither nondelipidated nor delipidated embryos that received transplanted LDs developed beyond the eight-cell stage (Fig. S5A). In addition, LDs were degraded even when the LD-transplanted embryos were cultured with triacsin $\mathrm{C}$ and DEUP simultaneously, as in the case of triacsin C treatment alone (Fig. S5B). Again, these results suggest that ACSL activity is important not only for new LD formation, but also for their maintenance.

\section{DISCUSSION}

Although delipidation methods based on centrifugation have frequently been used in porcine oocytes and embryos, which contain high levels of LDs, this study is the first to adapt such a system to mouse oocytes with lower levels of LDs, and to isolate intact LDs from oocytes without cytoplasmic contamination. Our method enabled us to investigate the nature of LDs in detail during early embryonic development. We found that oocytes were fertilized and developed normally even after delipidation, and that LDs were newly synthesized through FA activation catalyzed by ACSLs, even in the absence of FAs in the cultured media. Inhibition of ACSL activity caused not only blockage of new LD synthesis but also a reduction in the number of pre-existing LDs, which led to developmental impairment. In addition, we showed that overabundance of LDs had a negative effect on embryonic development. These results suggest the importance of synthesis and maintenance of LDs, likely mediated ACSL activity, during preimplantation embryonic development.

One limitation of forced lipophagy, our previously established method for degradation of LDs, is that its efficiency depends on intrinsic autophagic activity (Tatsumi et al., 2018). Consequently, LDs might not be selectively degraded when autophagic activity is low. After fertilization, autophagy is strongly induced, but its activity is reduced during the first cleavage (from the late one- to early twocell stage), and reactivated subsequently (Tsukamoto et al., 2008). The precise mechanism of autophagy induction after fertilization remains unknown [although we previously revealed that fertilizationinduced autophagy is independent of mTORC1, which inactivates autophagy (Yamamoto et al., 2014)]; therefore, the level of forced lipophagy cannot be artificially controlled. As a consequence, LDs are not completely degraded after the induction of forced lipophagy and LD remnants may mask subsequent phenotypes.

To overcome the limitation, we developed an approach for removing LDs from mouse MII oocytes based on two-step centrifugation. This method enabled us to study embryonic development in the complete absence of LDs, and to demonstrate the existence of newly synthesized LDs. However, as with forced lipophagy, our developed method also has limitations.

First, although our new method is suitable for isolating almost all LDs from MII oocytes, it cannot efficiently remove LDs from fertilized embryos, including one- and two-cell embryos: in these contexts, small LDs are retained inside the cytoplasm even after two-step centrifugation (data not shown). The reason for these differences is currently unknown, but we speculate that LD morphology, as well as LD content at each developmental stage, might affect delipidation efficiency: LDs tend to be more clustered in MII oocytes relative to fertilized embryos with dispersed LDs (Bradley et al., 2016). Alternatively, considering that cytoplasmic LDs physically interact with other organelles, including mitochondria, lysosomes and the ER (Valm et al., 2017), and even the nucleus (Romanauska and Kohler, 2018), these associations may be tighter after fertilization, preventing LDs from being released outside the cytoplasm even after two-step centrifugation.

Second, our finding that the IVF success rate decreased nearly $50 \%$ after delipidation, whereas both ZP removal and ZP drilling increased this rate, suggested that delipidation, especially two-step centrifugation and/or hyperosmotic treatment, might change some feature of the ZP (e.g. induction of ZP hardening), resulting in inhibition of sperm-ZP penetration. However, we found that once the delipidated MII oocytes were fertilized by IVF or ICSI, their subsequent developmental potential was comparable with normal MII oocytes, indicating that delipidation did not affect oocyte competence. However, future studies into the developmental potential of embryos derived from delipidated MII oocytes are required, e.g. embryo transfer experiments.

Third, using the new method, we also successfully removed LDs from MII oocytes collected from different mouse strains, but the efficiency differed among strains: MII oocytes collected from B6 mice (used in this study) and $\mathrm{C} 3 \mathrm{H}$ mice were delipidated more efficiently than those of ICR mice (Fig. S1 and data not shown). This may be related to the difference in lipid metabolism (metabolic response) between mouse strains, as reported previously (Montgomery et al., 2017). In any case, additional efforts to optimize our technique, depending on each experimental condition, are required.

Why can embryos depleted of newly synthesized LDs develop to the eight-cell stage but not beyond that? In this study, we found that all delipidated and non-delipidated embryos cultured with triacsin $\mathrm{C}$ failed to develop beyond the eight-cell stage. Although this indicates that LD biogenesis does not make a major contribution to early embryonic development before the eight-cell stage, it seems likely that endogenous lipid or glycogen, or exogenous energy substrates such as pyruvate, lactate and amino acids in culture media, can partially compensate for loss of LDs during early embryonic development after delipidation. Alternatively, endogenous FAs, likely produced by lipolysis or lipophagy from TG stored in LDs, may be used as phospholipids (rather than as an energy source) to generate new cellular membrane during preimplantation embryonic development. Given that the membrane surface increases $33 \%$ and $74 \%$ between the oocyte and the two- and four-cell stages, respectively (Pratt and George, 1989), there may be an even larger expansion after the eight-cell stage. Another possibility is that under LD-depleted conditions, the increase in free FAs originating from de novo synthesis may compromise cellular integrity and become incorporated into cytotoxic lipid species, such as acylcarnitine (McCoin et al., 
2015). Thus, LDs could serve to sequester excess FAs into LDs to prevent lipotoxicity.

Which is more important as a source of newly synthesized LDs: intracellular or extracellular FAs? The origin of FAs in oocytes and embryos remains unresolved, but given our finding that BODIPYC12 (a long-chain fatty acid analogue) was rapidly taken up and mobilized into newly synthesized LDs (Fig. 4D and Fig. S3), extracellular FAs are likely to be the preferential source for new LD production. On the other hand, we observed that LDs were newly synthesized even when delipidated MII oocytes were cultured in BSA- and FA-free conditions (Fig. S2), suggesting that endogenous FAs may contribute to newly synthesized LDs.

Our study raises an important question: how is LD content maintained during early embryonic development? Considering that LD biogenesis is strongly induced immediately after delipidation and that the new LDs accumulate stably, it is reasonable to consider the existence of mechanism(s) that regulate the balance between LD synthesis (TG storage) and LD lipolysis (TG hydrolysis). Hypothetically, during normal development, hydrolysis (lipase) activity could be exceptionally high to prevent overabundance of LDs (to keep LD amounts constant), whereas under LD-depleted conditions, newly synthesized LDs are stored stably, likely owing to a reduction in lipase activity, leading to restoration of $\mathrm{LD}$ content to the proper level. Our observation that non-delipidated embryos cultured with DEUP following LD transplantation, which led to unusual LD accumulation, tended to decrease their developmental ability, whereas no such decrease was observed in delipidated embryos, supports the 'balance' hypothesis. Although almost 50\% of the non-delipidated embryos still developed into blastocysts when cultured with DEUP following LD transplantation (Fig. 6C,D), those embryos exhibited a delay in development before the morula stage (data not shown), indicating that LD overabundance may influence cleavage speed (cell cycles) during early embryonic development. It will be interesting to examine this issue in future studies.

In addition, a recent study revealed the existence of $L D$ size-based degradation machinery in hepatocytes (Schott et al., 2019): larger LDs are preferentially degraded by lipases, whereas smaller (newly synthesized) LDs are consumed by lipophagy, a selective turnover of LDs by autophagy. Given our findings that a number of tiny new LDs appeared soon after delipidation, such small (non-clustered) LDs may be protected against degradation by cellular lipases, and therefore accumulate stably throughout embryonic development.

We also found that triacsin $\mathrm{C}$ treatment of non-delipidated embryos caused massive degradation of pre-existing LDs, and this degradation was not prevented by the pharmacological inhibition of lipase activity (Fig. S5B). These results suggest that ACSL activity was more important for both LD synthesis and their maintenance, as has been reported in hepatocytes (Fujimoto et al., 2007). Consistent with this, we found that the mRNA levels of Acsl family genes were differentially expressed in MII oocytes (Acsl3 mRNA was very abundant among the family), and that inhibition of ACSL enzymatic activity by triacsin $\mathrm{C}$ caused severe embryonic developmental defects. Therefore, we speculate that ACSL activity (predominantly ACSL3) is a key regulator of $\mathrm{LD}$ biogenesis during preimplantation embryonic development. The precise role of ACSLs needs to be further addressed.

Finally, given that maternal high-fat diet and obesity negatively affect oocyte quality, leading to impaired preimplantation development, alterations of epigenetic modification and defects in fetal development (Han et al., 2018), it is tempting to speculate that the protein and lipid composition of LDs might be changed by a high-fat environment. Consistent with this idea, we found that a high-fat diet leads to an increase in delipidation efficiency (R.A. and S.T., unpublished), implying a relationship between dietary fat and LD content in MII oocytes. Given that our new method can successfully isolate LDs from MII oocytes with high purity, it could be applied to proteomics and lipidomics analysis of the isolated LDs. Such an intensive study would shed some light on the functional link between LD biogenesis and oocyte quality during early embryonic development.

\section{MATERIALS AND METHODS \\ Mice}

Mice used in this study were 8- to 12-week-old C57BL/6J mice (Japan SLC), except for C3H mice (Japan SLC) and ICR mice (CLEA Japan) used for the experiment described in Fig. S1. Mice were fed a standard diet (MBR-1, Funabashi Farm) and maintained under specific pathogen-free conditions. All animal experiments were performed in accordance with the relevant guidelines and were approved by the Animal Care and Use Committee of the National Institute of Quantum and Radiological Science and Technology.

\section{Delipidation for MII oocytes and LD transplantation}

MII oocytes from superovulated females were collected as previously described (Tsukamoto et al., 2013). For delipidation, MII oocytes from superovulated females were incubated in FHM medium (270 mOsm) containing $10 \mu \mathrm{g} / \mathrm{ml}$ cytochalasin B (C2743, Sigma-Aldrich) and supplemented with either fatty acid-free BSA $(4 \mathrm{mg} / \mathrm{ml}$, A8806, SigmaAldrich; hereafter, FHM FA-free BSA) or polyvinylpyrrolidone ( $2 \mathrm{mg} / \mathrm{ml}$, PVP360, Sigma-Aldrich; hereafter, FHM-PVP), and then treated with hyaluronidase (H4272, Sigma-Aldrich) to remove cumulus cells. After several washes with FHM FA-free BSA or FHM-PVP, 50-70 MII oocytes were transferred to a sterile $1.5 \mathrm{ml}$ tube (72.692.210, SARSTEDS) containing $500 \mu \mathrm{l}$ of FHM FA-free BSA or FHM-PVP, and initially centrifuged for $10 \mathrm{~min}$ at $4200 \mathrm{~g}$ to aggregate intracellular LDs in the cell periphery (Fig. 1A). After initial centrifugation, an equal amount of prewarmed hyperosmotic FHM (630 mOsm, prepared by adding sucrose to FHM) was immediately added to the tube, yielding a final osmolality of $\sim 440 \mathrm{mOsm}$; mixed gently by pipetting; and subjected to secondary centrifugation for $10 \mathrm{~min}$ at $9500 \mathrm{~g}$ to release aggregated LDs into the expanded perivitelline (PV) space (Fig. 1A). All centrifugation steps were performed at room temperature. After the second centrifugation, MII oocytes were collected and observed individually to assess oocyte death and delipidation efficiency (Fig. 1C). LDs released into the perivitelline space were removed using a micropipette (inner diameter $\sim 15 \mu \mathrm{m}$ ) and a piezo micromanipulator (PMM-150FU, PrimeTech and PiezoXpert, Eppendorf). Osmolality was measured using an OSMOMAT 030 (Gonotec). Culture medium was produced according to a published protocol (Kito et al., 2004). We used FA-free BSA to minimize the contribution of BSA-derived lipids to the embryo cultures, with the exception of the experiments shown in Fig. 2C and Fig. S2, in which PVP was used instead of FA-free BSA.

For LD transplantation, a cluster of LDs isolated from MII oocytes were divided into two pieces, and each half was injected into individual blastomeres of non-delipidated or delipidated two-cell embryos (Fig. 6A and Movie 4) that had been treated with FHM FA-free BSA containing cytochalasin $\mathrm{B}$ for $30 \mathrm{~min}$ prior to the LD transplantation. After LD transplantation, these two-cell embryos were washed and further cultured under the indicated conditions in KSOM supplemented with FA-free BSA.

\section{IVF and ICSI, and embryo culture}

For in vitro fertilization (IVF), MII oocytes were incubated in cytochalasin B-free HTF supplemented with FA-free BSA (HTF with FA-free BSA), and then inseminated with sperm that had been capacitated for $1-2 \mathrm{~h}$ in TYH supplemented with FA-free BSA. Embryos were considered to be fertilized when a second polar body and two pronuclei were observed 6-7 $\mathrm{h}$ after insemination, and were cultured in KSOM supplemented with FA-free BSA until the blastocyst stage. To dissolve the ZP of MII oocytes, MII oocytes with or without delipidation were briefly incubated ( 1 min) 
in acidic Tyrode's solution [136.9 mM NaCl, $2.68 \mathrm{mM} \mathrm{KCl}, 2.16 \mathrm{mM}$ $\mathrm{CaCl}_{2}, 0.49 \mathrm{mM} \mathrm{MgCl} \cdot 6 \mathrm{H}_{2} \mathrm{O}, 5.55 \mathrm{mM}$ glucose, $4 \mathrm{mg} / \mathrm{ml} \mathrm{PVP}$ ( $\mathrm{pH} 2.5$ ), $278 \mathrm{mOsm}]$. The resulting ZP-free MII oocytes were cultured for $30 \mathrm{~min}$ prior to IVF in HTF with FA-free BSA and then inseminated as described above. When necessary, a microhole was drilled into the ZP of the MII oocyte (ZP drilling) using a piezo micromanipulator just prior to IVF.

Intracytoplasmic sperm injection (ICSI) was carried out according to a published protocol (Yoshida and Perry, 2007) with some modifications. Sperm collected from the cauda epididymis was cultured for 30-45 min in a $100 \mu \mathrm{l}$ drop of TYH (with FA-free BSA) covered with mineral oil, and the sperm suspension was mixed with FHM medium containing $12 \%$ polyvinylpyrrolidone. Individual sperm heads were separated by applying a few piezo pulses, and then injected into an MII oocyte with or without delipidation using a piezo micromanipulator. After ICSI, surviving MII oocytes were maintained in KSOM supplemented with FA-free BSA. Fertilized oocytes with two pronuclei and a second polar body were selected and cultured until the blastocyst stage.

For time-lapse observation (Movies 2 and 3), embryos were cultured in $40 \mu \mathrm{l}$ of KSOM supplemented with FA-free BSA in a LinKID micro25 dish (Dai Nippon Printing). Images were captured at 15 min intervals using a real-time cultured cell monitoring system (CCM-MULTI, ASTEC) equipped with a $10 \times$ objective lens (Plan, NA0.25, WD 10.5, Nikon).

In some experiments, embryos were cultured with $5 \mu \mathrm{M}$ triacsin $\mathrm{C}$ (an ACSL inhibitor, 10007448, Cayman Chemical) or with $100 \mu \mathrm{M}$ diethylumbelliferyl phosphate (DEUP, a lipase inhibitor, D7692, Sigma), which were dissolved in DMSO (D2650, Sigma-Aldrich). Embryos were cultured with the indicated chemicals from the one-cell stage $(6-7 \mathrm{~h}$ after IVF) until the DMSO-treated embryos had reached the blastocyst stage (96-98 $\mathrm{h}$ after IVF). LD-transplanted embryos were cultured with the indicated chemicals from the two-cell stage (24-26 h after IVF). Embryo culture was performed at $37^{\circ} \mathrm{C}$ under low-oxygen condition $\left(90 \% \mathrm{~N}_{2}, 5 \%\right.$ $\mathrm{O}_{2}, 5 \% \mathrm{CO}_{2}$ ).

\section{Fluorescence microscopy}

To visualize individual organelles, live oocytes or embryos were incubated with BODIPY 493/503 (1-2 $\mu \mathrm{g} / \mathrm{ml}$, D3922, ThermoFisher Scientific), LysoTracker Red Lysosomal Probe ( $2 \mu \mathrm{M}$, PA-3015, Lonza Walkersville), MitoTracker Red CMXRos (40 nM, M7512, ThermoFisher Scientific) or ER-Tracker Red ( $2 \mu \mathrm{M}, \mathrm{E} 34250$, ThermoFisher Scientific) in PB1 FA-free BSA for 30-60 min, washed once and transferred to PB1 FA-free BSA in a glass-bottom dish (D11140H, Matsunami Glass) covered with mineral oil. Images were acquired using a spinning disk confocal (X-Light V2, Molecular Devices) set up on an inverted microscope (IX83, Olympus) using a $63 \times$ Plan APO 1.4 NA oil immersion objective (Olympus), a CCD camera (Prime 95B, Photometrics) and LaserLED Hybrid fluorescence (X-Cite TURBO, Excelitas Technologies). The microscope apparatus was controlled using the MetaMorph software (Molecular Devices). All fluorescence images were acquired in a single plane, except for those in Fig. 6E, in which $z$-stack images are shown (10 sections, $2 \mu \mathrm{m}$ steps).

For imaging of BODIPY 558/568 C12, MII oocytes/embryos were incubated with $10 \mu \mathrm{M}$ BODIPY 558/568 C12 (D3835, ThermoFisher Scientific; $20 \mathrm{mM}$ BODIPY 558/568 C12 stock solution in DMSO was diluted in PB1 FA-free BSA) for 30 min, washed several times with PB1 FA-free BSA and immediately observed using the X-Light V2 spinning disk confocal as described above, except that a Tokai Hit Stage Top incubator was attached to the microscope. Time-lapse images were acquired every $2 \mathrm{~min}$ for 30 or $60 \mathrm{~min}$ (Fig. S3A,B). In some experiments, after staining with BODIPY 558/568 C12, MII oocytes or embryos were further incubated with BODIPY 493/503 for $30 \mathrm{~min}$, to visualize both the earliest steps of LD formation and mature LDs. When necessary, embryos were co-cultured with the indicated inhibitors.

For quantification of cellular LDs, the area of BODIPY 493/503-stained LDs was manually quantified from three independent experiments using the MetaMorph software. The lower fluorescence intensity threshold was chosen such that BODIPY 493/503-stained LDs appeared as single and nonclustered circular particles (ranging from 0.1 to $5 \mu \mathrm{m}$ in diameter), and the resultant area was measured. Identical thresholds were applied to all experimental conditions.

\section{Electron microscopy}

Electron microscopy was performed as described previously (Tsukamoto et al., 2013). The number of individual LDs with single and non-clustered morphology was counted manually, and the area of interest was measured using ImageJ (NIH) and imported into Microsoft Excel. The number of analyzed oocytes/embryos $(n)$ is indicated in each figure.

\section{TG analysis}

TG species analysis was conducted in MII oocytes maintained in FHM-PVP medium. One-hundred MII oocytes, with or without delipidation, were washed several times with PBS supplemented with PVP, transferred into a sterile microtube containing $20 \mu \mathrm{l}$ Ultrapure water (43001-1B, Kanto Chemical) and stored at $-80^{\circ} \mathrm{C}$ until use. Sample preparation and liquid chromatography/Fourier transform-type mass spectrometry (LC/FT-MS) analysis was performed at the Environmental Technology Department at the Chemicals Evaluation and Research Institute, Japan (CERI, Tokyo). The details of sample preparation and LC/FT-MS analysis have been described previously (Namekawa et al., 2018).

\section{Quantitative real-time PCR}

Total RNA from 30-40 MII oocytes was extracted using CellAmp Direct RNA Prep Kit (Takara) and subjected to real-time PCR using One Step TB Green PrimeScript RT-PCR Kit III (Takara). Real-time PCR was performed with a Thermal Cycler Dice Real Time System (Takara). The relative expression ratio was calculated using the comparative $\mathrm{Ct}$ method, and $\mathrm{Actb}$ ( $\beta$-actin) was used for normalization. Primers for Acsl1 (MA175328, Takara), Acsl3 (MA169187, Takara), Acsl4 (MA108448, Takara), Ascl5 (MA057264, Takara), Acsl6 (MA115382, Takara) and Actb (MA050368, Takara) were used.

\section{Statistical analysis}

Statistical significance was analyzed using two-tailed Student's $t$-test for normally distributed data sets, the Mann-Whitney $U$-test for two nonparametric data sets, and one-way ANOVA with Tukey's multiple comparison test when more than two groups were analyzed. All analyses were performed in Prism 6 (GraphPad). Data represent either means \pm s.e.m. or means \pm s.d., as indicated in the figure legends. Significance levels: n.s., non-significant, $* P<0.05, * * P<0.01$, $* * * P<0.001$, $* * * * P<0.0001$.

\section{Acknowledgements}

We thank Yasutomo Kubota and Jun Kudo (Molecular Devices, Japan) for advice on imaging analysis, Yosuke Ishihara (Tokai EMA) for EM analysis, Hidenori Yamanaka and Seto Ryoya (CERI, Tokyo) for TG analysis, and Ayako Wada and Tomomi Hatakeyama for excellent technical assistance.

\section{Competing interests}

The authors declare no competing or financial interests.

\section{Author contributions}

Conceptualization: R.A., S.T.; Methodology: R.A., S.T.; Validation: R.A., M.I., T.T., A.Y., K.S., S.I., N. Minami, S.T.; Investigation: R.A., M.I., T.T., S.T.; Data curation: R.A.; Writing - original draft: S.T.; Writing - review \& editing: R.A., M.I., T.T., A.Y., T.K., N. Miyasaka, K.S., S.I., N. Minami; Supervision: S.T.; Project administration: S.T.; Funding acquisition: N. Minami, S.T.

\section{Funding}

N. Minami was supported by the Japan Society for the Promotion of Science (KAKENHI program; $19 \mathrm{H} 03136$ and 16H05042). S.T. was supported by the Japan Agency for Medical Research and Development (16gk0110015h0001), the Japan Science and Technology Agency (ERATO program, MIZUSHIMA Intracellular Degradation; JPMJER1702), the joint research program of the Institute for Molecular and Cellular Regulation, Gunma University (18009) and the Takeda Science Foundation.

\section{Supplementary information}

Supplementary information available online at

http://dev.biologists.org/lookup/doi/10.1242/dev.181925.supplemental 


\section{References}

Bickel, P. E., Tansey, J. T. and Welte, M. A. (2009). PAT proteins, an ancient family of lipid droplet proteins that regulate cellular lipid stores. Biochim. Biophys. Acta 1791, 419-440. doi:10.1016/j.bbalip.2009.04.002

Bradley, J. and Swann, K. (2019). Mitochondria and lipid metabolism in mammalian oocytes and early embryos. Int. J. Dev. Biol. 63, 93-103. doi:10. $1387 / \mathrm{ijdb}$.180355ks

Bradley, J., Pope, I., Masia, F., Sanusi, R., Langbein, W., Swann, K. and Borri, P. (2016). Quantitative imaging of lipids in live mouse oocytes and early embryos using CARS microscopy. Development 143, 2238-2247. doi:10.1242/dev.129908

Brasaemle, D. L., Dolios, G., Shapiro, L. and Wang, R. (2004). Proteomic analysis of proteins associated with lipid droplets of basal and lipolytically stimulated 3T3L1 adipocytes. J. Biol. Chem. 279, 46835-46842. doi:10.1074/jbc.M409340200

Buhman, K. K., Chen, H. C. and Farese, R. V.Jr. (2001). The enzymes of neutra lipid synthesis. J. Biol. Chem. 276, 40369-40372. doi:10.1074/jbc.R100050200

Cermelli, S., Guo, Y., Gross, S. P. and Welte, M. A. (2006). The lipid-droplet proteome reveals that droplets are a protein-storage depot. Curr. Biol. 16 1783-1795. doi:10.1016/j.cub.2006.07.062

Dunning, K. R., Cashman, K., Russell, D. L., Thompson, J. G., Norman, R. J. and Robker, R. L. (2010). Beta-oxidation is essential for mouse oocyte developmental competence and early embryo development. Biol. Reprod. 83 909-918. doi:10.1095/biolreprod.110.084145

Dunning, K. R., Russell, D. L. and Robker, R. L. (2014). Lipids and oocyte developmental competence: the role of fatty acids and beta-oxidation Reproduction 148, R15-R27. doi:10.1530/REP-13-0251

Fujimoto, Y., Itabe, H., Sakai, J., Makita, M., Noda, J., Mori, M., Higashi, Y., Kojima, S. and Takano, T. (2004). Identification of major proteins in the lipid droplet-enriched fraction isolated from the human hepatocyte cell line $\mathrm{HuH} 7$. Biochim. Biophys. Acta 1644, 47-59. doi:10.1016/j.bbamcr.2003.10.018

Fujimoto, Y., Itabe, H., Kinoshita, T., Homma, K. J., Onoduka, J., Mori, M., Yamaguchi, S., Makita, M., Higashi, Y., Yamashita, A. et al. (2007) Involvement of ACSL in local synthesis of neutral lipids in cytoplasmic lipid droplets in human hepatocyte HuH7. J. Lipid Res. 48, 1280-1292. doi:10.1194/jlr M700050-JLR200

Fujino, T., Kang, M.-J., Suzuki, H., lijima, H. and Yamamoto, T. (1996). Molecular characterization and expression of rat acyl-CoA synthetase 3. J. Biol. Chem. 271 16748-16752. doi:10.1074/jbc.271.28.16748

Gardner, D. K. and Leese, H. J. (1986). Non-invasive measurement of nutrient uptake by single cultured pre-implantation mouse embryos. Hum. Reprod. 1 25-27. doi:10.1093/oxfordjournals.humrep.a136336

Han, L., Ren, C., Li, L., Li, X., Ge, J., Wang, H., Miao, Y.-L., Guo, X., Moley, K. H., Shu, W. et al. (2018). Embryonic defects induced by maternal obesity in mice derive from Stella insufficiency in oocytes. Nat. Genet. 50, 432-442. doi:10.1038/ s41588-018-0055-6

Herms, A., Bosch, M., Ariotti, N., Reddy, B. J. N., Fajardo, A., Fernández-Vidal, A., Alvarez-Guaita, A., Fernández-Rojo, M. A., Rentero, C., Tebar, F. et al. (2013). Cell-to-cell heterogeneity in lipid droplets suggests a mechanism to reduce lipotoxicity. Curr. Biol. 23, 1489-1496. doi:10.1016/j.cub.2013.06.032

Hillman, N. and Flynn, T. J. (1980). The metabolism of exogenous fatty acids by preimplantation mouse embryos developing in vitro. J. Embryol. Exp. Morphol. 56, 157-168

Ichimura, Y., Kumanomidou, T., Sou, Y.-S., Mizushima, T., Ezaki, J., Ueno, T., Kominami, E., Yamane, T., Tanaka, K. and Komatsu, M. (2008). Structura basis for sorting mechanism of p62 in selective autophagy. J. Biol. Chem. 283 22847-22857. doi:10.1074/jbc.M802182200

Igal, R. A. and Coleman, R. A. (1996). Acylglycerol recycling from triacylglycerol to phospholipid, not lipase activity, is defective in neutral lipid storage disease fibroblasts. J. Biol. Chem. 271, 16644-16651. doi:10.1074/jbc.271.28.16644

Kang, M.-J., Fujino, T., Sasano, H., Minekura, H., Yabuki, N., Nagura, H., lijima, H. and Yamamoto, T. T. (1997). A novel arachidonate-preferring acyl-CoA synthetase is present in steroidogenic cells of the rat adrenal, ovary, and testis Proc. Natl. Acad. Sci. USA 94, 2880-2884. doi:10.1073/pnas.94.7.2880

Kassan, A., Herms, A., Fernández-Vidal, A., Bosch, M., Schieber, N. L., Reddy, B. J. N., Fajardo, A., Gelabert-Baldrich, M., Tebar, F., Enrich, C. et al. (2013) Acyl-CoA synthetase 3 promotes lipid droplet biogenesis in ER microdomains. J. Cell Biol. 203, 985-1001. doi:10.1083/jcb.201305142

Kito, S., Hayao, T., Noguchi-Kawasaki, Y., Ohta, Y., Hideki, U. and Tateno, S (2004). Improved in vitro fertilization and development by use of modified human tubal fluid and applicability of pronucleate embryos for cryopreservation by rapid freezing in inbred mice. Comp. Med. 54, 564-570.

Leese, H. J. and Barton, A. M. (1984). Pyruvate and glucose uptake by mouse ova and preimplantation embryos. Reproduction 72, 9-13. doi:10.1530/jrf.0.0720009

Mashek, D. G., Bornfeldt, K. E., Coleman, R. A., Berger, J., Bernlohr, D. A. Black, P., DiRusso, C. C., Farber, S. A., Guo, W., Hashimoto, N. et al. (2004) Revised nomenclature for the mammalian long-chain acyl-CoA synthetase gene family. J. Lipid Res. 45, 1958-1961. doi:10.1194/jlr.E400002-JLR200

McCoin, C. S., Knotts, T. A. and Adams, S. H. (2015). Acylcarnitines-old actors auditioning for new roles in metabolic physiology. Nat. Rev. Endocrinol. 11, 617-625. doi:10.1038/nrendo.2015.129
Men, H., Spate, L. D., Murphy, C. N. and Prather, R. S. (2015). Cryopreservation of in vitro-produced early-stage porcine embryos in a closed system. Biores. Open Access 4, 258-265. doi:10.1089/biores.2015.0012

Montgomery, M. K., Brown, S. H. J., Mitchell, T. W., Coster, A. C. F., Cooney, G. J. and Turner, N. (2017). Association of muscle lipidomic profile with high-fat diet-induced insulin resistance across five mouse strains. Sci. Rep. 7, 13914 doi:10.1038/s41598-017-14214-1

Nagashima, H., Kashiwazaki, N., Ashman, R. J., Grupen, C. G., Seamark, R. F. and Nottle, M. B. (1994). Removal of cytoplasmic lipid enhances the tolerance of porcine embryos to chilling. Biol. Reprod. 51, 618-622. doi:10.1095/biolreprod51. 4.618

Nagashima, H., Kashiwazaki, N., Ashman, R. J., Grupen, C. G. and Nottle, M. B (1995). Cryopreservation of porcine embryos. Nature 374, 416. doi:10.1038/ $374416 \mathrm{a0}$

Namekawa, J., Yasui, M., Katayanagi, A., Shirai, M. and Asai, F. (2018). Increased hepatic triglyceride level induced by a glucokinase activator in mice. Fundam. Toxicol. Sci. 5, 13-20. doi:10.2131/fts.5.13

Pol, A., Gross, S. P. and Parton, R. G. (2014). Review: biogenesis of the multifunctional lipid droplet: lipids, proteins, and sites. J. Cell Biol. 204, 635-646. doi:10.1083/jcb.201311051

Pratt, H. P. M. and George, M. A. (1989). Organisation and assembly of the surface membrane during early cleavage of the mouse embryo. Roux Arch. Dev. Biol. 198, 170-178. doi:10.1007/BF02438942

Rambold, A. S., Cohen, S. and Lippincott-Schwartz, J. (2015). Fatty acid trafficking in starved cells: regulation by lipid droplet lipolysis, autophagy, and mitochondrial fusion dynamics. Dev. Cel/32,678-692. doi:10.1016/j.devcel.2015. 01.029

Romanauska, A. and Kohler, A. (2018). The inner nuclear membrane is a metabolically active territory that generates nuclear lipid droplets. Cell 174 700-715.e718. doi:10.1016/j.cell.2018.05.047

Schott, M. B., Weller, S. G., Schulze, R. J., Krueger, E. W., Drizyte-Miller, K., Casey, C. A. and McNiven, M. A. (2019). Lipid droplet size directs lipolysis and lipophagy catabolism in hepatocytes. J. Cell Biol. 218, 3320-3335. doi:10.1083/ jcb.201803153

Tatsumi, T., Takayama, K., Ishii, S., Yamamoto, A., Hara, T., Minami, N., Miyasaka, N., Kubota, T., Matsuura, A., Itakura, E. et al. (2018). Forced lipophagy reveals that lipid droplets are required for early embryonic development in mouse. Development 145, dev161893. doi:10.1242/dev.161893

Tauchi-Sato, K., Ozeki, S., Houjou, T., Taguchi, R. and Fujimoto, T. (2002). The surface of lipid droplets is a phospholipid monolayer with a unique fatty Acid composition. J. Biol. Chem. 277, 44507-44512. doi:10.1074/jbc.M207712200

Tomoda, H., Igarashi, K. and Omura, S. (1987). Inhibition of acyl-CoA synthetase by triacsins. Biochim. Biophys. Acta 921, 595-598. doi:10.1016/00052760(87)90088-9

Tsukamoto, S., Kuma, A., Murakami, M., Kishi, C., Yamamoto, A. and Mizushima, N. (2008). Autophagy is essential for preimplantation development of mouse embryos. Science 321, 117-120. doi:10.1126/science.1154822

Tsukamoto, S., Hara, T., Yamamoto, A., Ohta, Y., Wada, A., Ishida, Y., Kito, S. Nishikawa, T., Minami, N., Sato, K. et al. (2013). Functional analysis of lysosomes during mouse preimplantation embryo development. J. Reprod. Dev. 59, 33-39. doi:10.1262/jrd.2012-096

Valm, A. M., Cohen, S., Legant, W. R., Melunis, J., Hershberg, U., Wait, E., Cohen, A. R., Davidson, M. W., Betzig, E. and Lippincott-Schwartz, J. (2017) Applying systems-level spectral imaging and analysis to reveal the organelle interactome. Nature 546, 162-167. doi:10.1038/nature22369

Vessey, D. A., Kelley, M. and Warren, R. S. (2004). Characterization of triacsin C inhibition of short-, medium-, and long-chain fatty acid: CoA ligases of human liver J. Biochem. Mol. Toxicol. 18, 100-106. doi:10.1002/jbt.20009

Walther, T. C. and Farese, R. V.Jr. (2012). Lipid droplets and cellular lipid metabolism. Annu. Rev. Biochem. 81, 687-714. doi:10.1146/annurev-biochem061009-102430

Watanabe, T., Thayil, A., Jesacher, A., Grieve, K., Debarre, D., Wilson, T., Booth, M. and Srinivas, S. (2010). Characterisation of the dynamic behaviour of lipid droplets in the early mouse embryo using adaptive harmonic generation microscopy. BMC Cell Biol. 11, 38. doi:10.1186/1471-2121-11-38

Welte, M. A. (2015). Expanding roles for lipid droplets. Curr. Biol. 25, R470-R481. doi:10.1016/j.cub.2015.04.004

Yamamoto, A., Mizushima, N. and Tsukamoto, S. (2014). Fertilization-induced autophagy in mouse embryos is independent of mTORC1. Biol. Reprod. 91, 7. doi:10.1095/biolreprod.113.115816

Yoneda, A., Suzuki, K., Mori, T., Ueda, J. and Watanabe, T. (2004). Effects of delipidation and oxygen concentration on in vitro development of porcine embryos. J. Reprod. Dev. 50, 287-295. doi:10.1262/jrd.50.287

Yoshida, N. and Perry, A. C. F. (2007). Piezo-actuated mouse intracytoplasmic sperm injection (ICSI). Nat. Protoc. 2, 296-304. doi:10.1038/nprot.2007.7

Zechner, R., Madeo, F. and Kratky, D. (2017). Cytosolic lipolysis and lipophagy: two sides of the same coin. Nat. Rev. Mol. Cell Biol. 18, 671-684. doi:10.1038/nrm. 2017.76 